\title{
EL PROCESO CONSTITUYENTE VENEZOLANO
}

\author{
Ricardo Combellas*
}

I.- Este momento es estelar para el futuro de Venezuela, en la medida en que se está decidiendo la modalidad y definitiva convocatoria de una asamblea nacional constituyente, que por decir lo menos, redefina el marco institucional de la República. La decisión constituyente ha arribado a un estadio que le impide dar marcha atrás. No sólo la propuesta gana adeptos entre un sector abrumadoramente mayoritario de los venezolanos, sino que asumida como bandera electoral por el candidato y hoy Presidente Hugo Chávez Frías, su abrumador triunfo es el mejor aval de la ahora y definitiva bandera nacional.

II.- La retrospectiva histórica revela que la convocatoria constituyente es la culminación de la primera etapa del proceso constituyente iniciado en el país con los dolorosos sucesos de fines de febrero del año 1989. A partir de entonces el paradigma constitucional sufrió los embates de la "vitrina rota" del 27 de febrero, y comenzó a deteriorarse paulatinamente, de tal forma que la decisión constituyente hoy se torna irreversible. En su momento (vid. el libro Una Constitución para el Futuro, publicado el año 1994), resumí los factores que a mi entender habían quebrantado el paradigma constitucional, y lo hacían por ende vulnerable, frágil, siempre amenazado de reemplazo. Ellos son:

1) El carácter programático-ideológico de la Constitución no se asumió con la fuerza necesaria para su concreción. La desviación populista, el clientelismo y la corrupción desvirtuaron el programa constitucional.

2) Además, el programa constitucional no fue acompañado, o lo fue retardada, incompleta y reactivamente, por una acción legislativa eficaz y proyectiva que lo impulsara de forma coherente.

3) En tercer lugar, el Estado no diseñó las adecuadas garantías institucionales para la mejor proyección de los derechos fundamentales. El gravísimo deterioro de la calidad de vida de amplios estratos de la población (dedo acusador de una Constitución social retórica), y los balances en rojo que consecutivamente arrojan las violaciones de los derechos humanos, constituyen el mayor mentís de la carta de derechos establecida en la Constitución.

4) El deterioro del Poder Judicial, garante por excelencia del Estado de Derecho, ha impedido su efectiva realización, y por consiguiente desvirtuado el principio central de la supremacía constitucional y la autonomía de las ramas del Poder Público.
5) El pálido desarrollo del principio democrático recogido por la Constitución, y su desviación partidocrática, tutelar y cerrada a la participación, ejemplifica en el país una suerte de régimen político que Maurice Duverger denominó alguna vez como "democracia sin el pueblo".

A partir de 1989 se desarrolla un período dilemático construido en torno a la opción reforma general versus asamblea constituyente, que en su líneas fundamentales intentaré resumir:

El año 1989 es el año de la aparición de la Comisión Bicameral de Revisión Constitucional del Congreso de la República. Comienza por proponer el procedimiento de la enmienda, para luego desembocar, dada la amplitud que adquiere su agenda propositiva, en la reforma general de la Constituyente. El año de 1992, luego del fallida rebelión militar del 4 de febrero, paradójicamente triunfante como movimiento de opinión deslegitimador del gobierno de Pérez, fue el año del aparente apogeo y comienzo de la declinación del proyecto de la Bicameral. Efectivamente, cuando aparecía como la única alternativa institucional viable para relegitimar el modelo político vigente, naufragó ante la rutina parlamentaria que se apoderó de sus más caros propósitos. Retomado el año 1994, luego del triunfo de Rafael Caldera en las elecciones del diciembre de 1993, en una muestra descarada de ausencia de voluntad política y "gatopardismo", el Congreso de la República enterró definitivamente la reforma general de la Constitución.

III.- La propuesta constituyente tiene su hito primigenio, y por tanto de indiscutible trascendencia histórica, en los manifiestos que a partir del 30 de junio de 1990 publicó en la prensa nacional el denominado Frente Patriótico, liderado por Manuel Quijada, Juan Liscano y Manuel Alfredo Rodríguez. Su argumentación fundamental se centró en que el proyecto político que había dado origen a la Constitución se encontraba agotado, desvalorizado por el hegemonismo partidista y sus consecuencias en el manejo corrupto de la cosa pública y desnacionalización de las riquezas del país entregadas a otros países, y la profunda crisis moral, política, económica y social que agobiaba a Venezuela.

Ex-Presidente de la Comisión Presidencial para la Reforma del Estado y Director Ejecutivo de la Comisión Presidencial Constituyente de Venezuela. 
A partir de la rebelión militar del 4 de febrero despertó un abanico rico en propuestas constituyentes, incluido el Movimiento Bolivariano Revolucionario 200, liderado por Hugo Chávez y sus compañeros comandantes de la rebelión, pero también por significativos sectores de la sociedad civil, grupos de juristas, y la voz de la provincia, donde resaltó el para entonces gobernador del Estado Zulia, Oswaldo Alvarez Paz, que en su calidad de candidato presidencial del partido COPEI, enarbolaría la propuesta constituyente como bandera de su campaña electoral, el año señalado de 1993.

El debate constituyente influyó y fue recepcionado por la Comisión Bicameral, al incluir ésta a la constituyente como un instrumento de revisión profunda de las estructuras del Estado, como quedó estampado en su articulado así como en su justificación explicativa en la Exposición de Motivos, que merece aquí dada su patente actualidad, citarse textualmente:

"Pero de la mayor importancia es la previsión de que se pueda convocar a una Asamblea Constituyente, que a juicio de la Comisión tendría el poder originario, en el caso de que el pueblo así lo decidiere. Se establece de esta manera un mecanismo para que sin romper la continuidad jurídica del Estado venezolano, se pueda llegar a decidir, si el pueblo así lo considera necesario, la renovación total de la Carta Fundamental y el funcionamiento y estructura de los Poderes Públicos. Por supuesto, y aun cuando se sostiene que la Asamblea Constituyente dentro de su soberanía podría tomar a este respecto las decisiones que quisiera, se establece el límite de un año para su funcionamiento y se señala el precepto de que si se dicta una nueva Constitución, debe ser sometida después a referéndum popular. Se adoptan las medidas necesarias para la composición de la Asamblea, para evitar las dificultades que podrían surgir si esto se dejara a criterio del legislador".

IV. Una frase del general De Gaulle en los difíciles días del tránsito de la IV a la V República francesa vale la pena aquí citar: "Cuando los acontecimientos hablan muy claro y se está de acuerdo en el fondo, los procedimientos tienen una flexibilidad considerable".

Los acontecimientos hablaron claro el pasado 6 de diciembre, y la inmensa mayoría de los venezolanos está de acuerdo en el fondo, un clamor irresistible de cambio a canalizar gracias a la pronta convocatoria de una asamblea nacional constituyente, primera etapa conclusiva del proceso constituyente que se inició en el país, como señalamos supra, a fines de febrero de 1989.

Los procedimientos, en definitiva las formas jurídicas, por supuesto que son importantes, pero a todas luces secundarios frente al hecho político fundamental: el llamado al poder constituyente originario, con el fin de abrir cauces al rediseño institucional de la República. Lo que deseo transmitir lo expresan con claridad meridiana las palabras del destacado jurista alemán, Martin Kriele: "La fuerza explosiva que se halla en la idea de la soberanía del pueblo no puede ser eliminada por ningún Estado constitucional; sólo puede ser moderada y suavizada".

Los poderes constituidos (el Presidente, la Corte Suprema de Justicia, el Congreso), afincados en el respeto mutuo implicado en el principio de colaboración de las ramas del Poder Público, deben atender solícita y prontamente este llamado, que no es otro que el llamado al titular de la soberanía democrática, el pueblo, para que legitime con su sí aprobatorio la convocatoria constituyente. Por supuesto que el respaldo de la iniciativa popular es un corolario fundamental para alcanzar el objetivo propuesto.

Entonces comienza el formidable desafío: la elaboración ciudadana de la nueva Constitución de la República. Digo ciudadana adrede, pues a diferencia de nuestra restrictiva y elitista tradicional forma de hacer constituciones, ésta debe contar, si se quiere responder con éxito al ingente desafío, con una amplia y decisiva participación de la sociedad civil en todos los pasos de su elaboración. Tamaño reto, pues la vieja cultura política que nos rige, golpeada pero no muerta, conspira con su talante desmovilizador y cupular, en menoscabo de tan trascendental tarea.

Algunos requisitos, de entrada, considero ineludibles para garantizar el éxito de la faena a emprender. En primer lugar, la conciencia de que la participación en el proceso de elaboración de la nueva Constitución no debe ser excluyente sino, muy por el contrario, incluyente de todos los sectores de la vida nacional. Pero esto no es suficiente, y merece por tanto una aclaratoria: cierto que la constituyente debe orientarse por el consenso, pero no cualquier consenso, sino un consenso constructivo, es decir, aquel que llene las profundas aspiraciones y expectativas de cambio de la sociedad venezolana. El consenso no puede pactar con el pasado, y menos con una forma tradicional de hacer política, abrumadoramente rechazada por el común de los ciudadanos.

Asimismo, es necesario de una vez dejar sentado que la constituyente no puede ser "embozalada", por la sencilla razón, elemental doctrina constitucional, de que los poderes constituidos no pueden poner límites al poder constituyente. La Carta Magna se abrirá, no debemos tener complejo en asumirlo, desde el primer artículo al último, con el propósito de introducir todas las modificaciones que se juzguen convenientes. Magno es entonces el verdadero desafío. Como reza el antiguo refrán castellano: "Cada mochuelo a su olivo". Ha llegado el tiempo en que cada cual esté en su puesto (el puesto constituyente), cumpliendo con su deber.

El año 1994, cuando se presentó el conflicto afortunadamente superado, entre el Presidente y el Congreso en torno a los alcances de sus respectivas competencias en la situación de emergencia económica en ese tiempo surgida, consecuencia de la grave crisis bancaria que azotó con fuerza al país, y ante la eventual consulta refrendaria con que había amagado el Presidente, un periodista solicitó mi opinión, sobre lo cual en pocas palabras respondí: el referéndum puede ser catalogado de extraconstitucional (recuérdese el carácter excesivamente representativo de la Constitución de 1961), pero nunca de inconstitucional.

Las razones que justificaron mi lacónica respuesta fueron brevemente las siguientes: primero, el referéndum es una institución profundamente democrática, en la medida en que se consulta al pueblo directamente, como titular de la soberanía, asuntos de trascendencia nacional; segundo, es democrático no sólo únicamente por la razón señalada, sino también porque se respetan las garantías que protegen los 
derechos y libertades fundamentales de la persona humana, y de manera especial por sus implicaciones para la decisión popular, las libertades de expresión, información, reunión y manifestación. Este aspecto establece una diferencia radical entre el referéndum y el plebiscito consustancial a los gobiernos de cariz autoritario.

En suma, el referéndum, independientemente de su configuración jurídica, tiene un valor político innegable y poderoso, que las ramas del Poder Público del Estado deben recepcionar e interpretar de tal forma que asuma inmediatamente eficacia jurídica.

La actual realidad venezolana expresa esa tensión política y jurídica: existe un clamor evidente de cambio, que sólo puede ser canalizado en paz y democráticamente con la convocatoria de una asamblea nacional constituyente. Como señala la sentencia del 24 de noviembre de 1990 de la Corte Suprema de Justicia colombiana, que facilitó el llamado popular a la constituyente: "El clamor popular para que ocurra el fortalecimiento institucional es un hecho público y notorio que en derecho no requiere prueba".

Actualmente todos los caminos, sea su base de sustentación constitucional o legal, conducen al referéndum. Constituye un deber de naturaleza profundamente democrática, darle respuesta rápida al anhelo ciudadano, que lo alienta y reclama. Es la oportunidad de la voluntad política del pueblo decidiendo sobre el futuro de su propia existencia democrática. Bien recoge en estas palabras su sentido el constitucionalista español Antonio Torres del Moral: "Como trasunto directo de la soberanía, cuanta mayor y más directa sea la intervención del pueblo en su ejercicio, tanta mayor fuerza democrática alcanza a la Constitución y al régimen que instaura".

Abramos las compuertas de la incomprensión que atenazan los tradicionales privilegios partidocráticos, y permitamos que el pueblo exprese su voluntad constituyente por cauces pacíficos y democráticos. Es nuestro deber, es nuestra responsabilidad.

\section{ADENDA: LA CONSTITUYENTE ORIGINARIA}

Desde principios de los años noventa, y de manera especial el año 1992, está planteado en Venezuela el debate sobre la naturaleza de la constituyente que nos toca convocar. En términos de propuestas normativas, la constituyente originaria fue recogida por la comisión bicameral que presidió Rafael Caldera, y la constituyente derivada por el grupo de estudios constitucionales dirigido por Allan Brewer-Carías. Por supuesto que ya el año 1990 el Frente Patriótico había lanzado al país un programa constituyente originario, lo que fue rafirmado por el Movimiento Bolivariano Revolucionario 200, liderado por Hugo Chávez y los comandantes de la rebelión militar del 4 de febrero. El que esto escribe nunca tuvo dudas sobre la naturaleza originaria del proceso constituyente venezolano, tal como lo plasmé a lo largo de estos años en libros, folletos, entrevistas y artículos de opinión.

La constituyente que la abrumadora mayoría de los venezolanos (no abrigo duda de ello), vamos a convocar el próximo 25 de abril, es originaria, tal como lo explicita el decreto dictado por el Presidente Chávez, a tenor de su iniciativa refrendaria, concretada el pasado 2 de febrero. Valgan pues estas notas para clarificar a los ciudadanos de qué constituyente estamos entonces hablando:

Primeramente, en cuanto a los precedentes históricos, la constituyente revolucionaria de 1947 lo fue originaria, así como la reciente colombiana de 1991. Por cierto, el Derecho Constitucional clásico no concebía el poder constituyente sino como originario, y entendía al constituyente derivado como una contradicción, por la sencilla razón de que lo que constituye no puede ser constituido, prefiriendo en consecuencia para este supuesto el concepto de asamblea o convención constitucional. No obstante, el uso y la doctrina recepcionaron con el tiempo la novedosa terminología, sobre todo a partir de la posguerra, cuando las constituciones comenzaron a prescribir constituyentes derivadas, como poder de reforma (amending power, pouivoir constituant institué, poder de revisión de la constitución). A todas estas, qué distingue a una de la otra:

En primer lugar, la constituyente originaria no tiene límites temáticos, todo lo puede, a diferencia de la derivada, cuya agenda es en principio prefijada por el poder constituido. Ilustración de esta última lo fue la constituyente argentina de 1994, cuya agenda fue previamente elaborada por el Acuerdo de Olivo, suscrito por los dos grandes partidos, el Justicialista y el Radical.

En segundo lugar, su poder tiene un vigor y una jerarquía superiores al de los poderes establecidos, cuyas competencias puede restringir, e incluso, razón extrema, revocar y hasta disolver. Como bien expresó el fallecido Alvaro Gómez Hurtado, copresidente de la constituyente colombiana, con palabras patéticas el sentido de este poder: omnipotente y omnímodo. Muy por el contrario, la consituyente derivada se concentra en la elaboración y aprobación de una constitución, no entrando en relaciones, ni de cooperación pero tampoco de conflicto, con los poderes establecidos.

Por último, los actos promulgados por la constituyente originaria, sea cual sea su tenor y alcance, no están sujetos a control jurisdicional alguno, por la sencilla razón de que su voluntad es política, expresión de la soberanía popular; que crea y modifica a su antojo el ordenamiento jurídico. Esta nota definitoria explica el porqué del referéndum aprobatorio de la nueva Constitución: los constituyentes responden así de sus actos ante el soberano popular, su base de legitimación.

Por supuesto que el poder constituyente originario no es absoluto, al estar limitado por tres factores: su base democrática, los derechos fundamentales (anteriores y superiores a cualquier configuración temporal de poder), y las condiciones existenciales del Estado (territorio, población y principio soberano), amén de que como realidad histórico-concreta, está condicionado por las normas supranacionales y el derecho internacional.

Los venezolanos rechazamos una constituyente derivada, bautizada en el argot popular como "bobalicona", "zanahoria" y "chucuta", al igual que una que abra el camino al poder despótico, arbitrario y autoritario. Sí ansiamos un cambio profundo, que transforme radicalmente el sistema político y rediseñe la institucionalidad republicana. Esto sólo lo puede afrontar una constituyente originaria. Bienvenida entonces, y éxito a su ingente labor. 


\section{RESUMEN}

La Asamblea Nacional Constituyente permitirá redefinir el marco institucional de la república venezolana. Este proceso constituyente iniciado en 1989 aborda una etapa definitiva debido al apoyo mayoritario de los venezolanos y a que ha sido asumido como bandera por el hoy presidente Hugo Chávez.

El paradigma constitucional ha tenido tropiezos originados por el populismo, el clientelismo, la corrupción, la falta de un trabajo legislativo oportuno, la falta de garantías a los derechos fundamentales, el deterioro del poder judicial y la falta de una participación política abierta.

Para llevar a cabo una asamblea nacional constituyente es fundamental la participación amplia que lleve a un consenso constructivo. Es decir, se tratará de iniciar el 25 de abril una constituyente originaria que implica que su capacidad decisoria no esté sujeta a ningún poder establecido, ya que su origen es la expresión política de la soberanía popular que va a modificar el ordenamiento jurídico existente.

Palabras clave: Asamblea Nacional Constituyente, participación, soberanía popular, Constitución, consenso.

\section{ABSTRACT}

The National Assembly Constituency will permit the redefinition of the institutional framework of the Venezuelan Republic. This fundamental process started in 1989 outlines a definitive stage due to the major support of the Venezuela people, and has been assumed as the platform of present president Hugo Chávez.

The constitutional paradigm has flaws that originate from the following: populism, clientelism, corruption, the lack of timely legislative work, the lack of guarantees to basic rights, the deterioration of judicial power, and the lack of open political participation.

For the National Assembly Constituency to work, extensive participation is fundamental to bring forth constructive consensus. As its origins now is the political expression of popular sovereignty that modifies the existing judicial order, it wil try to iniciate on the $25^{\text {th }}$ of April a new constituency that implies that its capacity for decision making is not subject to any establishe power.

Key words: National Assembly Constituency, participation, popular sovereignty, Constitution, consensus.

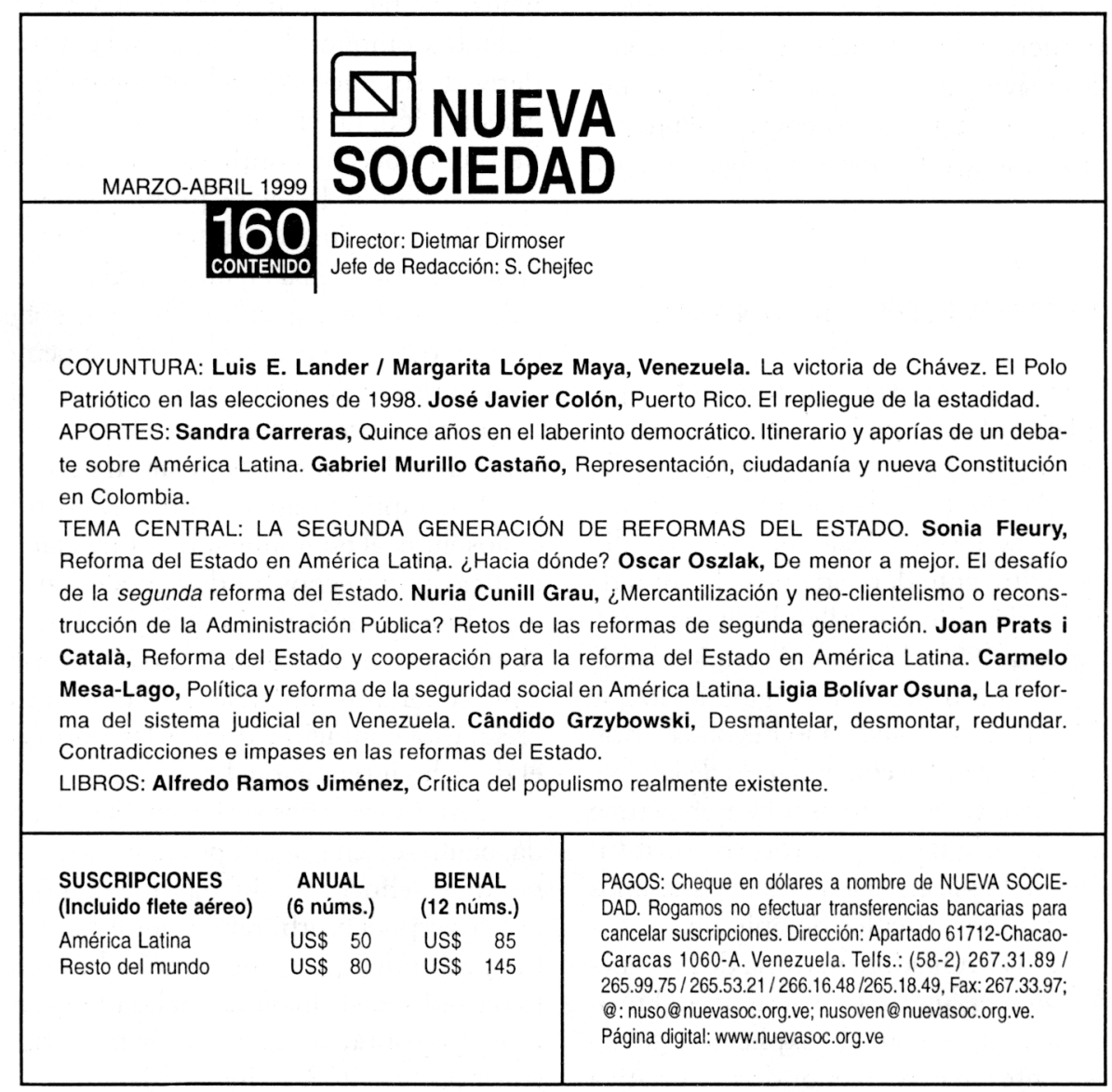

